\title{
Combined Metabolic Activators decrease liver steatosis by activating mitochondrial metabolism in a Golden Syrian hamster study
}

Hong Yang ${ }^{1, \#}$, Jordi Mayneris-Perxachs ${ }^{2,3 \#,}$ Noemí Boqué4, Josep M del Bas ${ }^{4}$, Lluís Arola ${ }^{4,5}$, Meng Yuan ${ }^{1}$, Hasan Turkez ${ }^{6}$, Mathias Uhlén ${ }^{1}$, Jan Borén ${ }^{7}$, Cheng, Zhang ${ }^{1,8}$, Adil Mardinoglu ${ }^{1,9 *}$ Antoni Caimari ${ }^{*}$

${ }^{1}$ Science for Life Laboratory, KTH - Royal Institute of Technology, Stockholm, Sweden.

${ }^{2}$ Department of Diabetes, Endocrinology and Nutrition, Hospital Universitari de Girona Doctor Jospe Trueta and Girona Biomedical Research Centre (IDIBGI), Girona, Spain.

${ }^{3}$ CIBER Pathophysiology of Obesity and Nutrition (CIBEROBN), Instituto de Salud Carlos III, Madrid, Spain.

${ }^{4}$ Eurecat, Centre Tecnològic de Catalunya, Technological Unit of Nutrition and Health, Reus, Spain.

${ }^{5}$ Universitat Rovira i Virgili, Nutrigenomics Research Group; Department of Biochemistry and Biotechnology, Campus Sescelades, Tarragona, Spain.

${ }^{6}$ Department of Medical Biology, Faculty of Medicine, Atatürk University, Erzurum, Turkey ${ }^{7}$ Department of Molecular and Clinical Medicine, University of Gothenburg and Sahlgrenska University Hospital, Gothenburg, Sweden.

${ }^{8}$ School of Pharmaceutical Sciences, Zhengzhou University, Zhengzhou, PR China

${ }^{9}$ Centre for Host-Microbiome Interactions, Faculty of Dentistry, Oral \& Craniofacial Sciences, King's College London, London, United Kingdom.

*Correspondence: Dr. Adil Mardinoglu (adilm@scilifelab.se), Dr. Antoni Caimari (antoni.caimari@eurecat.org).

\# These authors contributed equally to this work. 


\begin{abstract}
The prevalence of non-alcohol fatty liver disease (NAFLD), defined as the liver's excessive fat accumulation, continues to increase dramatically. We have recently revealed the molecular mechanism underlying NAFLD using in-depth multi-omics profiling and identified that combined metabolic activators (CMA) could be administered to decrease the amount of hepatic steatosis (HS) in mouse model and NAFLD patients based on systems analysis. Here, we investigated the effects of a CMA including L-carnitine, N-acetyl-1-cysteine, nicotinamide riboside and betaine on a Golden Syrian hamster NAFLD model fed with HFD, and found that HS was decreased with the administration of CMA. To explore the mechanisms involved in the clearance HS, we generated liver transcriptomics data before and after CMA administration, and integrated these data using liver-specific genome-scale metabolic model of liver tissue. We systemically determined the molecular changes after the supplementation of CMAs and found that it activates mitochondria in the liver tissue by modulating the global fatty acid, amino acids, antioxidant and folate metabolism.
\end{abstract}




\section{Introduction}

Hepatic steatosis (HS) is defined as the excessive accumulation of fat in the liver $(>5.5 \%$ tissue weight), and it is the most common liver disease worldwide ${ }^{1}$. Non-alcoholic fatty liver disease (NAFLD), a widespread metabolic disorder, refers to a group of conditions including HS and various degrees of liver inflammation such as non-alcoholic steatohepatitis (NASH). NAFLD can progress to cirrhosis, and ultimately hepatocellular carcinoma (HCC) which are much more severe liver diseases ${ }^{1,2,3}$. It has been reported that the global prevalence of NAFLD is approximately at $\sim 25 \%{ }^{1,4,5}$. Although research in drug development for NAFLD is intense and advancing rapidly, there are still significant unmet challenges with no effective drug approved for this condition ${ }^{6}$.

The insufficient capacity to remove incomplete products of fatty acid oxidation is one of NAFLD's main hallmark ${ }^{7}$. Oxidative stress has a significant role in NAFLD's pathogenesis and is characterized by impaired function of electron transport chains, impaired oxidation and increased production of reactive oxygen species (ROS) ${ }^{8,9,10}$. Glutathione (GSH) is the most abundant endogenous antioxidant in response to oxidative stress and increased $\operatorname{ROS} 11,12,13$. Our previous studies, combing personalised genome-scale metabolic models (GEMs) and indepth multi-omics profiling based on clinical data indicated an augmented requirement for nicotinamide adenine dinucleotide $\left(\mathrm{NAD}^{+}\right)$and GSH in NAFLD patients. A three-step strategy including (i) activating mitochondrial fatty acid uptake, (ii) increasing fatty acid oxidation, (iii) increasing the availability of GSH can be employed to decrease of the HS in NAFLD patients $14,15,16,17,18$.

To validated our hypothesis, we performed a study in which supplementation of a CMA including serine (GSH precursor), nicotinamide riboside (NR, NAD ${ }^{+}$precursor) and $\mathrm{N}$-acetyl1-cysteine (NAC, GSH precursor) was given to mice fed a high-fat diet (HFD) for 14 days. The results showed that supplementation of CMA significantly decreased the amount of liver fat by promoting the fat oxidation in mitochondria in the liver ${ }^{15,19}$. We further studied the effect of 
the CMA including serine, NR, NAC and L-carnitine tartrate (LCT, salt form of 1-carnitine that promote the update of fat to mitochondria) in a one-day proof-of-concept human supplementation study (NCT03838822). We observed that the supplementation resulted in increased of fatty acid oxidation and de novo GSH synthesis ${ }^{20}$. Recently, we also tested the effect of the long-term effect of the CMA including serine, NR, NAC and LCT in 10 weeks randomised, placebo-controlled study and found that administration of CMA decreases the amount of HS in liver together with other clinical parameters including AST, ALT, uric acid and creatinine ${ }^{21}$. However, the underlying molecular mechanism associated with decreased HS has not been revealed in animal or human studies.

In this study, we investigated the effect of the supplementation of CMA, including NR, Lcarnitine, NAC, and betaine (as a GSH precursor since that can be converted to glycine and serine), in Golden Syrian hamsters with NAFLD induced by HFD. We used Golden Syrian hamsters since it is a better model for experimental studies on lipoprotein metabolism than other smaller-sized animals, e.g., rat and mouse ${ }^{22,23,24}$. Furthermore, we previously used this model to evaluate successfully the health effects of different bioactive compounds such as phytosterols ${ }^{25}$ and extracts of hazelnut skin ${ }^{26}$ and grape seed procyanidins ${ }^{25,27}$ against fatty liver, dyslipidaemia and adiposity. In the present study, we found that administration of CMA ameliorated HS. To explore the underlying molecular mechanisms involved in decreased HS, we generated liver transcriptomics data before and after supplementation of CMA and integrated these data using liver GEM. Finally, we systematically identified the phenotypical differences and presented the potential effects of CMA supplementation on the global metabolism based on systems analysis. 


\section{Results}

\section{CMA attenuate HFD-induced HS in a Golden Syrian hamster NAFLD model}

We fed Golden Syrian hamsters with either normal fat diet (NFD) or HFD for 8 weeks. Afterwards, we provided either a placebo or a CMA including NR, L-carnitine and NAC by la gavage and betaine in the drinking water to hamsters at intended human clinical doses (Dose I; HFD+MI_D1 group) and 2-fold (Dose II; HFD+MI_D2 group) dose levels for 4 weeks (Figure 1A). We assessed the changes in body weight, biometric and serum variables of each hamster group at week 8 and 12 and examined the phenotypic differences in all groups. We observed that the hamsters fed with HFD had significantly more severe grade of HS compared to those from the NFD-fed group ( $\mathrm{p}<0.05)$, and this HFD-induced HS was significantly attenuated in CMA-treated hamsters with Dose I and II (Figure 1B). Additionally, the histological analyses showed a significant increase of lipid deposition in the HFD group's liver compared to those of the control group (Figure 1C\&D). Specifically, the HFD groups developed microvesicular steatosis without apparent inflammation, sinusoidal dilatation or fibrosis (Figure 1D), and CMA supplementation with Dose I and II reduced the HFD-induced lipid deposition in the liver (Figure E\&F). The HFD-fed hamsters, compared to the control group, showed significantly increased liver weights $(\mathrm{p}=0.001)$ and relative mesenteric adipose tissue depots (MWAT\%, mean increase 37.5\%, p = 0.009) (Table 1). CMA treatment tended to induce a reduction in liver weight, mainly in hamsters supplemented with Dose II $(8.8 \%$ and $10.1 \%$ reduction with Dose I and II, respectively) compared to the HFD group. (Figure 1G). Body composition analysis revealed that HFD feeding led to a weight gain during the last 4 weeks of the study (mean increase 4.5\%, p = 0.03) (Figure 1H, Table 1). Hamsters treated by CMA with Dose II showed significantly $(\mathrm{p}=0.005)$ reduced body weight $(5.1 \%$ mean reduction $)$ (Figure $1 \mathrm{H}$, Table 1$)$ and significantly $(\mathrm{p}=0.05)$ increased lean/fat mass ratio $(10.9 \%$ mean increase) (Figure 1I, Table 1) between 8 weeks and 12 weeks. Furthermore, at the end of the study, the supplementation with Dose II significantly decreased body weight $(p=0.021)$ and body weight gain $(\mathrm{p}=0.0004)$ compared to the HFD-fed hamsters that received the placebo 
(Figure 1H, Table 1). This body weight loss could be attributed, at least in part, to the decreased cumulative caloric intake $(\mathrm{p}=0.009)$ displayed by the HFD+MI_D2 group during the 4-week CMA supplementation period when compared to the HFD group. A similar trend in body weight $(p=0.137)$ and body weight gain $(p=0.066)$ was observed in hamsters treated with Dose I in comparison with HFD group, although the differences were not significant (Figure $1 \mathrm{H}$, Table 1). We also measured the $\mathrm{NAD}^{+}$levels in the liver and found that its levels significantly increased with CMA Dose I and II supplementation compared to their HFD counterparts, as predicted in our earlier studies (Figure 1J).

We observed reduction in plasma triglyceride concentrations in CMA-treated hamsters $(16.5 \%$ and $28.7 \%$ mean reduction with Dose I and Dose II, respectively) (Table 1) than those in the HFD group in parallel to the decrease in HS. Additionally, we found that HFD significantly increased the plasma level of glucose $(p=0.02)$, total cholesterol $(\mathrm{CHOL} ; \mathrm{p}=0.0001)$, highdensity lipoprotein cholesterol (HDL-C; $\mathrm{p}=0.01$ ), and low-density lipoprotein cholesterol (LDL-C; $\mathrm{p}=0.001)$ during the 12 weeks feeding period (Table 1). We observed that CMA supplementation with different doses did not alter these parameters (Table 1).

\section{Transcriptomics alteration with HFD and CMA treatment}

To reveal the underlying molecular changes associated with the decreased HS after CMA treatment, we performed global transcriptomics analysis using RNA sequencing (RNA-seq) on liver tissue of each group. Globally, PCA revealed patterns of gene expression changes with HFD and CMA treatments (Figure 2A). At a 10\% false discovery rate (FDR), we found 3,317 differentially expressed genes (DEGs) between HFD and control group, 1,669 of which were significantly up-regulated and 1,648 of which were down-regulated (Figure 2B, Dataset S1). We performed KEGG pathway enrichment analysis on up-regulated and down-regulated DEGs separately in each group. Our results indicated that up-regulated DEGs were enriched in pathways including oxidative phosphorylation, ribosome, metabolic pathway, lysosome, cardiac muscle contraction, and spliceosome in HFD group vs control group (Figure 2C, 
Dataset S2). We found that fatty acid metabolism, catabolism of amino acids including branched-chain amino acids (BCAAs) and lysine, metabolism of amino acids (tryptophan, beta-alanine, glycine, serine, and threonine), as well as signalling pathways in the regulation of insulin resistance and fatty acid oxidation (e.g., AMPK-signalling pathway) were significantly enriched with the down-regulated DEGs in HFD group vs control group (Figure 2C, Dataset S2).

We also performed Gene Ontology (GO) enrichment analysis for the DEGs. Compared to the control group, we found that up-regulated genes in HFD group were most significantly enriched in nucleoside monophosphate metabolic process, ATP synthesis coupled electron transport, respiratory electron transport chain and purine nucleoside metabolic process. We also found that down-regulated genes were most significantly enriched in cellular protein modification process, protein modification process and cellular protein metabolic process in HFD vs control group (Dataset S2).

Next, we compared the gene expression changes between HFD and CMA-treated groups (fed with HFD + MI_D1 and HFD + MI_D2). In total, 80 (56 up-regulated and 24 down-regulated) and 641 DEGs (268 down-regulated and 373 up-regulated) (Figure 2D, Dataset S1) were identified in Dose I and II groups, respectively. We found that the log 2-fold changes in DEGs between two groups compared to HFD group are significantly positively correlated $(r=0.61$, $\mathrm{p}<2.2 \mathrm{e}-16$ ) (Figure 2E). This suggested that the supplementation with different dosages have a very similar effect on the hamster liver. Thus, we focused on Dose II group in the following analyses as it demonstrated more distinct and significant changes associated with the supplementation of CMA. The KEGG enrichment analysis showed that 14 pathways were upregulated after 4-week supplementation of CMA at Dose II (Figure 2C, Dataset S2). Of these, the most up-regulated pathways involved biosynthesis and metabolism of amino acids, including glycine, serine, threonine, tryptophan, alanine, aspartate glutamate, arginine and proline, as well as BCAAs (Figure 2C, Dataset S2). GO enrichment analysis of up-regulated 
DEGs showed that the oxoacid metabolic process, carboxylic acid metabolic process, and alpha-amino acid metabolic process are the most significantly altered biological processes (Dataset S2) in Dose II group vs HFD group.

\section{Reporter metabolites through the global analysis of transcriptomics data}

To evaluate the detailed metabolic differences in hamsters with or without CMA treatment upon HFD feeding, we constructed a liver-specific genomic-scale metabolic model for Golden Syrian hamster based on the Golden Syrian hamster orthologs of mouse genes in mouse metabolic reaction (MMR) ${ }^{28}$, and transcriptomics data by using INIT (Integrative Network Inference for Tissues) algorithm ${ }^{29}$ (Figure 3A). The constructed metabolic model, namely iHamsterHepatocyte1818, includes 3,867 metabolic reactions, 1,818 genes, and 3,205 metabolites.

Next, we performed reporter metabolite analysis to identify the key metabolic hubs in response to the supplementation of CMA with Dose II. We identified reporter metabolites using the differentially expression genes of Dose II vs HFD group and the network topology provided by iHamsterHepatocyte1818. Reporter metabolite analyses were used as statistical test to identify metabolites in the network for which a significant change occurred between the compared conditions. A total of 256 reporter metabolites (Reporter Features, p-value $<0.05$ ) for hamsters treated with CMA with Dose 2 were identified (Dataset S3). Of these, 143 and 113 reporter metabolites were associated with up-regulated and down-regulated genes in CMAtreated group, respectively. The association of the reporter metabolites with up- and downregulated genes and their metabolic subsystems classified in iHamsterHepatocyte1818 are presented (Figure 3B, C\&D). As shown in the figure, CMA treatment modulated several subsystems that are known to be associated with NAFLD, including amino acid metabolism (BCAAs, cysteine, methionine, glycine, serine, threonine, arginine, proline, alanine, aspartate, and glutamate), folate metabolism, tricarboxylic acid cycle (TCA) cycle (Figure 3B), fatty acid activation and oxidative phosphorylation (Figure 3C). In addition, CMA supplementation 
regulated the subsystems associated with biosynthesis and metabolism of cholesterol, biosynthesis and elongation of fatty acid, as well as nucleotide metabolism (Figure 3D).

\section{CMA boosts hepatic metabolism to attenuate HS}

Based on the integrative systems analysis, we found the CMA supplementation enhanced several NAFLD associated key metabolic pathways in the liver. We found that CMA supplementation, or more specifically, betaine supplementation, increased hepatic expression of genes involved in folate metabolism, including SHMT2, MTHFD1, DMGDH, SARDH and GNMT (Figure 4A\&B). This implicated an enhanced folate cycle in the liver. Besides, we observed a significant increase in the hepatic expression of $C B S$ and $C T H$ involved in transsulfuration pathway, where the supplemented NAC might play an important role (Figure 4A\&B). This may contribute to the GSH generation by synthesising cysteine and may play a crucial role in maintaining cellular redox homeostasis ${ }^{30}$.We also observed increased hepatic expression of key genes involved in mediating GSH metabolism, including GPX1, GPX4, GSTK1 and PRDX5 (Figure 4A\&B). Based on reporter metabolite analysis, we also identified betaine, dimethylglycine, sarcosine, 5,10-methylene-THF, 10-formyl-THF, THF, homocysteine, L-cystathionine and S-(formylmethyl) glutathione, which are associated with up-regulated genes in folate metabolism, transsulfuration pathway and glutathione metabolism (Figure 3B, Figure 4A\&B). Considering that one of the significant components of the CMA is betaine which is a precursor for these metabolic pathways (Figure 4A), these results suggested that the supplementation promoted the glutathione biosynthesis through the folate cycle and transsulfuration pathway.

Peroxisome and mitochondria co-operatively perform diverse metabolic processes, including fatty acid $\beta$-oxidation and cellular ROS homeostasis ${ }^{31}$. Short- and medium-chain fatty acids (SCFAs, MCFAs) are oxidized in the mitochondria whereas long-chain fatty acids (LCFAs) are oxidized in both mitochondria and peroxisome. Very-long-chain fatty acids (VLFAs) are preferentially oxidized in the peroxisomes ${ }^{32}$. We found that the hepatic expression of genes 
associated with fatty acid oxidation in peroxisome and mitochondria (SLC27A5, ABCD1, ACOT8, ACSM1, ACSM3 and ACSM5) were elevated in the liver of CMA-treated hamsters, which could be related to the supplementation of LCT and NR (Figure 4B\&C). Besides, we observed the genes involved in ROS metabolism including MPV17L, GSTK1, PRDX5, GPX1, and GPX4 (Figure 4B\&C), showed a significant increase which could be a result of the supplemented NR and elevated glutathione metabolism. SLC27A5, which facilitates the uptake of LCFAs, has been considered as long-chain and very-long-chain acyl-CoA (VLCFA-CoA) synthetases ${ }^{33,34}$. Interestingly, the hepatic expression of genes involved in de novo synthesis of $\mathrm{NAD}^{+}$, an important coenzyme for redox reactions, including TDO2, AFMID and KYNU were significantly increased after CMA supplementation. $\mathrm{NAD}^{+}$and $\mathrm{NADH}$ were also identified as reporter metabolites associated with upregulated genes in fatty acid oxidation, oxidative phosphorylation and TCA cycle (Figure 4B\&C). Notably, in agreement with the aforementioned findings, we found that $\mathrm{NAD}^{+}$level was significantly increased in the liver with CMA Dose I and II supplementation vs HFD (Figure 1J).

Additionally, we found that CMA supplementation increased the hepatic expression of genes involved in BCAAs catabolism, including BCAT2, BCKDHA, MCCC1, MCCC2, ALDH2, ALDH9A1 and PCCA (Figure 4B, Figure 5A). We also observed significantly enhanced hepatic expression of $I D H 2$ and $S D H B$ in TCA cycle. Increased TCA cycle and BCAAs catabolism could indicate that the fatty acid oxidation and cellular respiration are enhanced as suggested by a previous study ${ }^{35}$. Moreover, we observed that the hepatic expression of genes involved in arginine biosynthesis (GPT, GOT1, GPT2, CPS1 and ASS1) and genes involved in arginine and proline metabolism were significantly increased with the CMA supplementation. It has been reported that the downregulation of CPS1, the flux-generating urea cycle feeder enzyme, correlated with the loss of functional capacity for ureagenesis in patients with $\mathrm{NASH}^{36,37}$.

\section{Disscussion}

In this study, we evaluated the global changes induced by the CMA treatment (Figure 5B). We 
demonstrated that HFD-fed hamsters that received the CMA at both doses displayed an amelioration of hepatic steatosis compared to their HFD-fed control counterparts. This finding was accompanied by a significant decrease in final body weight and body weight gain in the hamsters supplemented with the Dose II, which can be attributed to the lower caloric intake observed in these animals. Interestingly, our transcriptomics analysis revealed that after CMA treatment there was a significant increase in the hepatic expression of genes in one-carbon metabolism and transsulfuration pathway, which are closely associated to the de novo GSH synthesis, which, in turn, is altered in patients with HS ${ }^{15}$. Moreover, we reported that CMA supplementation increased the expression of genes involved in BCAAs catabolism and urea cycle in the liver. Furthermore, we observed elevated $\mathrm{NAD}^{+}$hepatic levels, which implicated the enhancement of multiple metabolic pathways including fatty acids oxidation, the lack of which has a critical role in the development of NAFLD ${ }^{15,38}$, and strongly suggest CMA supplementation as a very promising strategy to ameliorate fatty liver.

Our findings agreed well with and also extended the predicted results from our recent work ${ }^{20}$. Our previous studies revealed that altered GSH and $\mathrm{NAD}^{+}$metabolism is a prevailing feature of NAFLD ${ }^{15}$. Amino acids, in particular serine, glycine, methionine, and several metabolites involved in one-carbon metabolism play crucial roles in NAFLD progression ${ }^{14}$. Mice with HFD-induced NAFLD also showed dysregulated one-carbon metabolism in liver $39,40$. Dysregulated BCAAs metabolism is significantly associated with the progression of NAFLD 41. The increased plasma level of BCAAs have been reported in NAFLD metabolomics studies 42. Based on our previous study, we predicted that catabolism of BCAAs was significantly increased after CMA supplementation in based on integration of GEMs and metabolomics data 20.

Serine hydroxymethyltransferase (SHMT) catalyses the interconversion of glycine and serine, which are precursors for the generation of GSH, an important antioxidant for maintaining the redox balance in fatty acid $\beta$-oxidation ${ }^{15,43}$. Glycine $N$-methyltransferase (GNMT), the main 
gene involved in liver S-adenosylmethionine (SAM) catabolism, is down-regulated in the liver of HFD hamsters ${ }^{44}$, as well as patients with cirrhosis and $\mathrm{HCC}^{45}$. In other studies, deletion of GNMT in mice led to excess increase in hepatic SAM, which is associated with fatty acid metabolism, oxidative stress and HS ${ }^{46,47}$. The down-regulation of $D M G D H$, which is a mitochondrial dimethylglycine dehydrogenase, was associated with the insulin resistance in a previous study ${ }^{48}$. Hepatic $C B S / C T H$ system was even positioned as a potential therapeutic target in NAFLD due to the deficiencies of $C B S$ and $C T H$ in rodents' study ${ }^{49,50}$. Taking into account the aforementioned findings ${ }^{51}$, in the present study, the increased expression of key genes involved in folate metabolism (SHMT2, MTHFD1, DMGDH, SARDH, and GNMT) and in the transsulfuration pathway $(C B S$ and $C T H)$ found in the livers of CMA-supplemented hamsters strongly suggest that CMA ameliorated NAFLD promoting glutathione biosynthesis through the activation of these two metabolic pathways.

$I D H 2$ is considered a unique enzyme in the regulation of mitochondrial ROS ${ }^{52}$. IDH2 knockout accelerates oxidative stress, lipid accumulation and HS in HFD-challenged mice, which were restored by promoting $I D H 2$ expression ${ }^{53}$. In the present study, we found that $I D H 2$ was upregulated in the livers of hamsters after CMA supplementation vs HFD. Relevantly, the ROS metabolism-related genes MPV17L and GSTK1 displayed the same pattern of expression than those observed for $I D H 2$. MPV17L, a transmembrane protein, has been implicated in the regulation of peroxisomal ROS metabolism ${ }^{54}$. MPV17L also prevents mitochondrial dysfunction and apoptosis through its antioxidant and antiapoptotic properties in vivo and in vitro ${ }^{55}$. GSTK1 is a highly conserved enzyme potentially involved in redox reaction and has a crucial role in protection against oxidative stress ${ }^{56}$. In addition, the expression of GPX1 and GPX4 genes, which codify for the glutathione peroxidase (GPx) family of enzymes, were also up-regulated in the hamsters that received the CMA treatment. GPx catalyses the reduction of $\mathrm{H}_{2} \mathrm{O}_{2}$ and lipid peroxides through the conversion of GSH to GSSG and thereby plays a crucial role in the protection of cells against oxidative damage. Patients with NASH had significantly lower levels of GPx activity ${ }^{57}$. Overall, our results strongly suggest that CMA would protect 
against NAFLD-associated oxidative stress, at least in part, through the up-regulation of the expression of these key genes involved in ROS metabolism.

The increased hepatic expression of genes associated with fatty acid oxidation in peroxisome and mitochondria (SLC27A5, ABCD1, ACOT8, ACSM1, ACSM3 and ACSM5) found in response to the supplementation with CMA suggest that this multi-ingredient would tackle NAFLD by boosting this catabolic pathway in both organelles. Different previous findings contribute to reinforce our hypothesis. Thus, a recent study showed that knockout of SLC27A5 lead to the disrupted lipid metabolism and redox balance in HCC cells both in vitro and in vivo 58. In patients with severe steatohepatitis and cirrhotic liver, the hepatic expression level of SLC27A5 were significantly decreased and inversely correlated with histological progression 59. ABCDI mediates the uptake of the VLCFAs across the peroxisome membrane and its loss of function results in defective $\beta$-oxidation of VLCFA and increased cellular oxidative stress $60,61$.

In conclusion, the supplementation for 4 weeks with a cocktail of metabolic activators (CMA) including L-carnitine, $\mathrm{N}$-acetyl-1-cysteine, nicotinamide riboside and betaine at 2 intended human clinical doses ameliorated HS in HFD-fed Golden Syrian hamsters. This health effect was accompanied by body weight loss and decreased caloric intake in the animals that received Dose II. To shed light on the metabolisms by which CMA exerted its effects, we performed liver transcriptomics analysis and analysed the data using GEMs. We observed that CMA supplementation significantly attenuated the HFD-induced HS by modulating the GSH and $\mathrm{NAD}^{+}$metabolisms, as well as promoting fatty acid oxidation, BCAAs catabolism and urea cycle and eventually activating mitochondria. Our findings provide extra evidence about the beneficial effects of a treatment based on these metabolic activators against NAFLD. 


\section{Methods and materials}

\section{Animal model}

The Animal Ethics Committee of the Technological Unit of Nutrition and Health of Eurecat (Reus, Spain) and the Generalitat de Catalunya approved all procedures (DAAM 10026). The experimental protocol complied with the ARRIVE guidelines, followed the 'Principles of laboratory animal care' and was carried out in accordance to the EU Directive 2010/63/EU for animal experiments. All animals were housed individually at $22{ }^{\circ} \mathrm{C}$ under a light/dark cycle of $12 \mathrm{~h}$ (lights on at 09:00 am) and were given free access to food and water.

Thirty-nine 10-week-old male Golden Syrian hamsters (Janvier Labs, Saint Berthevin, France) weighting 110-120 g were used. After an adaptation period of 1 week, hamsters were randomly assigned into two experimental groups fed with a NFD $(n=9,11 \%$ calories from fat; Envigo, Barcelona, Spain) or a HFD $(\mathrm{n}=30,23 \%$ calories from fat and $1 \mathrm{~g} / \mathrm{kg}$ cholesterol; Envigo, Barcelona, Spain) (Dataset S4) for 8 weeks. In a previous study, using very similar diets we demonstrated that this period was useful to induce fatty liver and hypercholesterolemia in hamsters ${ }^{25}$. Afterwards, HFD group was further randomly distributed into three subgroups: placebo, CMA at Dose I (200mg/kg LC, $200 \mathrm{mg} / \mathrm{kg}$ NAC, $200 \mathrm{mg} / \mathrm{kg}$ NR and $400 \mathrm{mg} / \mathrm{kg}$ betaine; HFD+MI_D1 group) and multi-ingredient at Dose II (400mg/kg LC, 400 mg/kg NAC, $400 \mathrm{mg} / \mathrm{kg}$ NR and $800 \mathrm{mg} / \mathrm{kg}$ betaine; HFD+MI_D2 group) for the last 4 weeks. LCT contained $68.2 \%$ of LC and, therefore, to reach the desired LC doses hamsters were supplemented with $293 \mathrm{mg} / \mathrm{kg}$ and $586 \mathrm{mg} / \mathrm{kg}$ of LCT for Dose I and Dose II, respectively. LCT, NAC and NR were daily diluted with low-fat condensed milk diluted 1:3 with water (vehicle) and orally given to the hamsters. Four days before the beginning of the treatments, the animals were trained to lick diluted low-fat condensed milk diluted 1:3 with water $(0.2 \mathrm{~mL})$ to ensure voluntary consumption. It was confirmed that each hamster fully ingested the daily dose of the corresponding treatment, which was given daily beween 8:30-10:00 am. Betaine was included in opaque bottles containg the drinking water $(4 \mathrm{~g} / \mathrm{L}$ and $8 \mathrm{~g} / \mathrm{L}$ fos Dose I and Dose II, respectively) and renewed three times per week. Considering an average hamster's weight 
of $125 \mathrm{~g}$, the doses of LCT, NAC and NR used were equivalent to the daily consumption of $1,918 \mathrm{mg}$ and $3,836 \mathrm{mg}$ of these metabolic activators for a $60-\mathrm{kg}$ human ${ }^{62}$, for the Dose I and Dose II, respectively. For betaine, the extrapolated daily intake using the same formula were 3,836 $\mathrm{mg}$ and 7,672 $\mathrm{mg}$. These dosages are considered acceptable and safe in a context of a multi-ingredient supplementation to tackle NAFLD ${ }^{19}$.

Body weight and food intake were recorded once per week, and food was renewed daily. At 12 weeks, all experimental animals were sacrificed under anaesthesia (pentobarbital sodium, 60 $\mathrm{mg} / \mathrm{kg}$ body weight) after $6 \mathrm{~h}$ of diurnal fasting. Blood was collected by cardiac puncture, and serum was obtained by centrifugation and stored at $-20{ }^{\circ} \mathrm{C}$ until analysis. The liver, soleus and gastrocnemius muscles, and white adipose tissue (WAT) depots (retroperitoneal (RWAT) and mesenteric (MWAT) depots) were rapidly removed, weighed, frozen in liquid nitrogen and stored at $-70{ }^{\circ} \mathrm{C}$ until analysis.

\section{Histological evaluation}

Morphometric analyses of tissues and steatosis of liver histology were described as before ${ }^{25}$. In general, the entire histological section (approximate area $2 \mathrm{~cm}^{2}$ ) of the liver were analysed according to Brunt's golden standard score, estimating the percentage of the area covered by fat droplets and using a scored from 0 and 3 ( 0 , null, 1 , when steatosis was detected in up to $30 \%$ of the area; 2 , when steatosis was observed in between 30 and $60 \%$ of the area; 3 , when steatosis was observed in more than $66 \%$ of the area).

\section{Body Composition Analyses}

Body composition were analysed by Nuclear magnetic resonance (NMR). Lean and fat mass analyses were performed at the end of weeks 8 and 12 using an EchoMRI-700 ${ }^{\circledR}$ device (Echo Medical Systems, L.L.C., Houston, TX, United States). The measurements were performed in duplicate. Data are expressed in relative values as a percentage of body weight (\%). Lean/fat mass ratio was also calculated. 


\section{Serum Analysis}

Enzymatic colorimetric assays were used for the analysis of glucose, total cholesterol and triglycerides (QCA, Barcelona, Spain), HDL-cholesterol and LDL/VLDL-cholesterol (Bioassay systems, California, USA). Serum insulin levels were analysed using a hamster insulin ELISA kit (MyBiosource, Bizkaia, Spain).

\section{${ }^{1} \mathrm{H}$ NMR analysis for $\mathrm{NAD}^{+}$determination}

For ${ }^{1} \mathrm{H}$ NMR analysis, the metabolite aqueous extracts obtained in the liver were reconstituted in $700 \mathrm{ul}$ of a solution containing trisilylpropionic acid (TSP) $(0.74 \mathrm{mM})$ dissolved in $\mathrm{D}_{2} \mathrm{O}$ phosphate buffer $(0.05 \mathrm{M})$. Samples were vortexed, homogenised for $5 \mathrm{~min}$ and centrifuged (15 min at $14000 \mathrm{xg}$ ). Finally, the redissolved extractions were transferred into $5 \mathrm{~mm}$ NMR glass tubes. ${ }^{1} \mathrm{H}$ NMR measurements were performed following the procedure described by Vinaixa et al. (2010) ${ }^{63}$.

\section{Transcriptomics and Gene Set Enrichment analysis}

Total RNA was extracted from the liver samples using TriPure reagent (Roche Diagnostic, Sant Cugat del Valles, Barcelona, Spain) according to the manufacturer's instructions, and used in the subsequent mRNA sample preparation for sequencing using NovaSeq $6000 \mathrm{~S} 1$ platform with the standard Illumina RNA-seq protocol. The RNA-seq was performed at National Genomics Infrastructure (NGI, Sweden) (project number P15763). Gene abundance (in both raw counts and transcripts per million) was quantified using the kallisto pipeline ${ }^{64}$ based on Golden Syrian hamster (Mesocricetus auratus) genome (version MesAur1.0.101). In order to validate the intra-group homogeneity, we first performed a principal component analysis (PCA). We filtered out the S105 sample in control group based on the results of PCA analysis. Next, we used DESeq2 package in $R^{65}$ to identify differentially expressed genes (DEGs) (adjusted $\mathrm{p}$ value $<0.1$ ). Gene Ontology and KEGG pathway analysis were performed separately on upregulated and downregulated genes using DAVID Bioinformatics Functional Annotation Tool ${ }^{66}$. Only 'biological process' categories enriched with a BH-corrected $P \leq 0.05$ 
were considered for GO category analysis.

\section{Metabolic model reconstruction and Reporter metabolite analysis}

To provide a resource for automated and semi-automated reconstruction of liver-specific GEMs for Golden Syrian hamster, we constructed iHamsterHepatocyte1818 based on the MMR databases, transcriptomics data, and INIT (Integrative Network Inference for Tissues) algorithm, which has been used to reconstruct the functional GEMs based on bulk transcriptomics data as well as 56 user-defined metabolic tasks, which are known to occur in cells/tissues ${ }^{67}$ and should be performed by the resulting metabolic model. A reporter metabolite analysis was performed using RAVEN toolbox implemented in MATLAB 2020a based on DEGs obtained as described above, together with iHamsterHepatocyte1818.

\section{Statistical analysis}

The continuous variables of biological assay were showed as mean \pm SD. Grubbs' test was used to detect outliers, which were discarded for subsequent analyses. Differences between two groups were analysed by $t$-test from the SciPy package. Differences in steatosis scores were detected using the Chi-squared test. Missing values were dropped before the analysis. Categorical variables were analysed using Chi-square test. Principal component analysis (PCA) was performed on transcriptomics data sets to explore the quality of data and detect possible outliers. All results were considered statistically significant at $p<0.05$. Statistical analyses were performed using Python 3.7 and $R$ (v4.0.3) was used for data visualisation. 


\section{Conflict of interest statement}

AM, JB and MU are the founder and shareholders of ScandiBio Therapeutics and they filed a patent application on the use of CMA to treat NAFLD patients. The other authors declare no conflict of interest.

\section{Acknowledgments}

We gratefully acknowledge the help of Yaiza Tobajas, Anna Antolín, Iris Triguero, Cristina Egea and Gertruda Chomiciute, who are laboratory technicians at the Technological Unit of Nutrition and Health of Eurecat, for their technical support. The author would like to thank NGI Sweden for generation of transcriptomics data. AM and HY acknowledge support from the PoLiMeR Innovative Training Network (Marie Skłodowska-Curie Grant Agreement No. 812616) which has received funding from the European Union's Horizon 2020 research and innovation programme. The computations were performed on resources provided by SNIC through Uppsala Multidisciplinary Center for Advanced Computational Science (UPPMAX) under Project SNIC 2020/16-69.

\section{Funding}

This work was financially supported by the Agency for Business Competitiveness of the Government of Catalonia (ACCIÓ) [TECCT11-1-0012]. This work was financially supported by the Centre for the Development of Industrial Technology (CDTI) of the Spanish Ministry of Science and Innovation under grant agreement: TECNOMIFOOD project (CER-20191010). This work was financially supported by ScandiBio Therapeutics and Knut and Alice Wallenberg Foundation.

\section{Author contributions}

A.M., A.C., M.U., and J.B. designed the study; J.M.P., N.B., J.M.B., and L.A. did the experiments; H.Y., M.Y., and C.Z. analyzed the transcriptomics data; H.Y. and C.Z. interpreted the data; H.Y. wrote the manuscript; and all authors helped revise the manuscript. 


\section{References}

1. Abeysekera KWM, et al. Prevalence of steatosis and fibrosis in young adults in the UK: a population-based study. Lancet Gastroenterol Hepatol 5, 295-305 (2020).

2. Huang DQ, El-Serag HB, Loomba R. Global epidemiology of NAFLD-related HCC: trends, predictions, risk factors and prevention. Nat Rev Gastroenterol Hepatol, (2020).

3. Mitra S, De A, Chowdhury A. Epidemiology of non-alcoholic and alcoholic fatty liver diseases. Transl Gastroenterol Hepatol 5, 16 (2020).

4. Bedogni G, Nobili V, Tiribelli C. Epidemiology of fatty liver: an update. World $J$ Gastroenterol 20, 9050-9054 (2014).

5. Younossi ZM, Koenig AB, Abdelatif D, Fazel Y, Henry L, Wymer M. Global epidemiology of nonalcoholic fatty liver disease-Meta-analytic assessment of prevalence, incidence, and outcomes. Hepatology 64, 73-84 (2016).

6. Lazarus JV, et al. NAFLD - sounding the alarm on a silent epidemic. Nat Rev Gastroenterol Hepatol 17, 377-379 (2020).

7. Parekh S, Anania FA. Abnormal lipid and glucose metabolism in obesity: implications for nonalcoholic fatty liver disease. Gastroenterology 132, 2191-2207 (2007).

8. Koliaki C, et al. Adaptation of hepatic mitochondrial function in humans with nonalcoholic fatty liver is lost in steatohepatitis. Cell Metab 21, 739-746 (2015).

9. Masarone M, et al. Role of Oxidative Stress in Pathophysiology of Nonalcoholic Fatty Liver Disease. Oxid Med Cell Longev 2018, 9547613 (2018).

10. Mansouri A, Gattolliat CH, Asselah T. Mitochondrial Dysfunction and Signaling in Chronic Liver Diseases. Gastroenterology 155, 629-647 (2018).

11. Winterbourn CC, Hampton MB. Thiol chemistry and specificity in redox signaling. Free Radic Biol Med 45, 549-561 (2008).

12. Soga $\mathrm{T}$, et al. Serum metabolomics reveals gamma-glutamyl dipeptides as biomarkers for discrimination among different forms of liver disease. J Hepatol 55, 896-905 (2011).

13. Lushchak VI. Glutathione homeostasis and functions: potential targets for medical interventions. J Amino Acids 2012, 736837 (2012).

14. Mardinoglu A, Agren R, Kampf C, Asplund A, Uhlen M, Nielsen J. Genome-scale metabolic modelling of hepatocytes reveals serine deficiency in patients with nonalcoholic fatty liver disease. Nat Commun 5, 3083 (2014).

15. Mardinoglu A, et al. Personal model-assisted identification of $\mathrm{NAD}(+)$ and glutathione metabolism as intervention target in NAFLD. Mol Syst Biol 13, 916 (2017). 
16. Suarez M, Boque N, Del Bas JM, Mayneris-Perxachs J, Arola L, Caimari A. Mediterranean Diet and Multi-Ingredient-Based Interventions for the Management of Non-Alcoholic Fatty Liver Disease. Nutrients 9, (2017).

17. Mardinoglu A, et al. An Integrated Understanding of the Rapid Metabolic Benefits of a Carbohydrate-Restricted Diet on Hepatic Steatosis in Humans. Cell Metab 27, 559571 e555 (2018).

18. Mardinoglu A, Uhlen M, Boren J. Broad Views of Non-alcoholic Fatty Liver Disease. Cell Syst 6, 7-9 (2018).

19. Mardinoglu A, Ural D, Zeybel M, Yuksel HH, Uhlen M, Boren J. The Potential Use of Metabolic Cofactors in Treatment of NAFLD. Nutrients 11, (2019).

20. Zhang C, et al. The acute effect of metabolic cofactor supplementation: a potential therapeutic strategy against non-alcoholic fatty liver disease. Mol Syst Biol 16, e9495 (2020).

21. Zeybel M, Mardinoglu; A, Schwenk; Cheng Zhang; Saeed Shoaie; Jens Nielsen; Mathias Uhlén; Jan Borén MZOAMAXLHYCFMABSMGGDUWKJM. Combined Metabolic Cofactor Supplementation Reduces Liver Fat in Nonalcoholic Fatty Liver Disease. Cell Metabolism (under review), (2021).

22. Bravo E CA, Calcabrini A, Ortu G. Why prefer the golden Syrian hamster (Mesocricetus auratus) to the Wistar rat in experimental studies on plasma lipoprotein metabolism?. Comparative Biochemistry and Physiology Part B: Comparative Biochemistry 107(2), (1994).

23. Zhang $\mathrm{Z}$, et al. Choosing hamsters but not rats as a model for studying plasma cholesterol-lowering activity of functional foods. Mol Nutr Food Res 53, 921-930 (2009).

24. Dillard A, Matthan NR, Lichtenstein AH. Use of hamster as a model to study dietinduced atherosclerosis. Nutr Metab (Lond) 7, 89 (2010).

25. Laos S, et al. Long-term intake of soyabean phytosterols lowers serum TAG and NEFA concentrations, increases bile acid synthesis and protects against fatty liver development in dyslipidaemic hamsters. Br J Nutr 112, 663-673 (2014).

26. Caimari A, et al. The intake of a hazelnut skin extract improves the plasma lipid profile and reduces the lithocholic/deoxycholic bile acid faecal ratio, a risk factor for colon cancer, in hamsters fed a high-fat diet. Food Chem 167, 138-144 (2015).

27. Caimari A, del Bas JM, Crescenti A, Arola L. Low doses of grape seed procyanidins reduce adiposity and improve the plasma lipid profile in hamsters. Int J Obes (Lond) 37, 576-583 (2013).

28. Mardinoglu A, et al. The gut microbiota modulates host amino acid and glutathione metabolism in mice. Mol Syst Biol 11, 834 (2015). 
29. Agren R, Bordel S, Mardinoglu A, Pornputtapong N, Nookaew I, Nielsen J. Reconstruction of genome-scale active metabolic networks for 69 human cell types and 16 cancer types using INIT. PLoS Comput Biol 8, e1002518 (2012).

30. Bin P, Huang R, Zhou X. Oxidation Resistance of the Sulfur Amino Acids: Methionine and Cysteine. Biomed Res Int 2017, 9584932 (2017).

31. Fransen M, Lismont C, Walton P. The Peroxisome-Mitochondria Connection: How and Why? Int J Mol Sci 18, (2017).

32. Hunt MC, Alexson SE. Novel functions of acyl-CoA thioesterases and acyltransferases as auxiliary enzymes in peroxisomal lipid metabolism. Prog Lipid Res 47, 405-421 (2008).

33. Steinberg SJ, Kemp S, Braiterman LT, Watkins PA. Role of very-long-chain acylcoenzyme A synthetase in X-linked adrenoleukodystrophy. Ann Neurol 46, 409-412 (1999).

34. Doege $\mathrm{H}$, et al. Targeted deletion of FATP5 reveals multiple functions in liver metabolism: alterations in hepatic lipid homeostasis. Gastroenterology 130, 1245-1258 (2006).

35. Lerin C, et al. Defects in muscle branched-chain amino acid oxidation contribute to impaired lipid metabolism. Mol Metab 5, 926-936 (2016).

36. De Chiara F, et al. Urea cycle dysregulation in non-alcoholic fatty liver disease. $J$ Hepatol 69, 905-915 (2018).

37. Eriksen PL, et al. Non-alcoholic fatty liver disease alters expression of genes governing hepatic nitrogen conversion. Liver Int 39, 2094-2101 (2019).

38. Covarrubias AJ, Perrone R, Grozio A, Verdin E. NAD $(+)$ metabolism and its roles in cellular processes during ageing. Nat Rev Mol Cell Biol, (2020).

39. Christensen KE, Wu Q, Wang X, Deng L, Caudill MA, Rozen R. Steatosis in mice is associated with gender, folate intake, and expression of genes of one-carbon metabolism. J Nutr 140, 1736-1741 (2010).

40. Pacana T, et al. Dysregulated Hepatic Methionine Metabolism Drives Homocysteine Elevation in Diet-Induced Nonalcoholic Fatty Liver Disease. PLoS One 10, e0136822 (2015).

41. Lake AD, et al. Branched chain amino acid metabolism profiles in progressive human nonalcoholic fatty liver disease. Amino Acids 47, 603-615 (2015).

42. Kalhan SC, et al. Plasma metabolomic profile in nonalcoholic fatty liver disease. Metabolism 60, 404-413 (2011). 
43. Nguyen D, Samson SL, Reddy VT, Gonzalez EV, Sekhar RV. Impaired mitochondrial fatty acid oxidation and insulin resistance in aging: novel protective role of glutathione. Aging Cell 12, 415-425 (2013).

44. Garcia AP, Palou M, Sanchez J, Priego T, Palou A, Pico C. Moderate caloric restriction during gestation in rats alters adipose tissue sympathetic innervation and later adiposity in offspring. PLoS One 6, e17313 (2011).

45. Avila MA, et al. Reduced mRNA abundance of the main enzymes involved in methionine metabolism in human liver cirrhosis and hepatocellular carcinoma. $J$ Hepatol 33, 907-914 (2000).

46. Varela-Rey M, et al. Fatty liver and fibrosis in glycine N-methyltransferase knockout mice is prevented by nicotinamide. Hepatology 52, 105-114 (2010).

47. Martinez-Una M, et al. S-Adenosylmethionine increases circulating very-low density lipoprotein clearance in non-alcoholic fatty liver disease. J Hepatol 62, 673-681 (2015).

48. Magnusson M, et al. Dimethylglycine Deficiency and the Development of Diabetes. Diabetes 64, 3010-3016 (2015).

49. Bravo E, et al. High fat diet-induced non alcoholic fatty liver disease in rats is associated with hyperhomocysteinemia caused by down regulation of the transsulphuration pathway. Lipids Health Dis 10, 60 (2011).

50. Sarna LK, Siow YL, O K. The CBS/CSE system: a potential therapeutic target in NAFLD? Can J Physiol Pharmacol 93, 1-11 (2015).

51. Maria Del Bas J, et al. Hepatic accumulation of S-adenosylmethionine in hamsters with non-alcoholic fatty liver disease associated with metabolic syndrome under selenium and vitamin E deficiency. Clin Sci (Lond) 133, 409-423 (2019).

52. Lee $\mathrm{JH}$, et al. Correction: Isocitrate dehydrogenase 2 protects mice from high-fat dietinduced metabolic stress by limiting oxidative damage to the mitochondria from brown adipose tissue. Exp Mol Med 52, 988 (2020).

53. Gong F, Gao L, Ding T. IDH2 protects against nonalcoholic steatohepatitis by alleviating dyslipidemia regulated by oxidative stress. Biochem Biophys Res Commun 514, 593-600 (2019).

54. Bonekamp NA, Volkl A, Fahimi HD, Schrader M. Reactive oxygen species and peroxisomes: struggling for balance. Biofactors 35, 346-355 (2009).

55. Krick S, et al. Mpv171 protects against mitochondrial oxidative stress and apoptosis by activation of Omi/HtrA2 protease. Proc Natl Acad Sci U S A 105, 14106-14111 (2008).

56. Blackburn AC, et al. Glutathione transferase kappa deficiency causes glomerular nephropathy without overt oxidative stress. Lab Invest 91, 1572-1583 (2011). 
57. Leach NV, et al. Serum homocysteine levels, oxidative stress and cardiovascular risk in non-alcoholic steatohepatitis. Eur J Intern Med 25, 762-767 (2014).

58. Gao Q, et al. SLC27A5 deficiency activates NRF2/TXNRD1 pathway by increased lipid peroxidation in HCC. Cell Death Differ 27, 1086-1104 (2020).

59. Enooku K, et al. Hepatic FATP5 expression is associated with histological progression and loss of hepatic fat in NAFLD patients. $J$ Gastroenterol 55, 227-243 (2020).

60. Singh I, Moser AE, Goldfischer S, Moser HW. Lignoceric acid is oxidized in the peroxisome: implications for the Zellweger cerebro-hepato-renal syndrome and adrenoleukodystrophy. Proc Natl Acad Sci U S A 81, 4203-4207 (1984).

61. Baarine M, Beeson C, Singh A, Singh I. ABCD1 deletion-induced mitochondrial dysfunction is corrected by SAHA: implication for adrenoleukodystrophy. $J$ Neurochem 133, 380-396 (2015).

62. Reagan-Shaw S, Nihal M, Ahmad N. Dose translation from animal to human studies revisited. FASEB J 22, 659-661 (2008).

63. Vinaixa $\mathrm{M}$, et al. Metabolomic assessment of the effect of dietary cholesterol in the progressive development of fatty liver disease. J Proteome Res 9, 2527-2538 (2010).

64. Bray NL, Pimentel H, Melsted P, Pachter L. Near-optimal probabilistic RNA-seq quantification. Nat Biotechnol 34, 525-527 (2016).

65. Love MI, Huber W, Anders S. Moderated estimation of fold change and dispersion for RNA-seq data with DESeq2. Genome Biol 15, 550 (2014).

66. Jiao X, et al. DAVID-WS: a stateful web service to facilitate gene/protein list analysis. Bioinformatics 28, 1805-1806 (2012).

67. Agren R, Mardinoglu A, Asplund A, Kampf C, Uhlen M, Nielsen J. Identification of anticancer drugs for hepatocellular carcinoma through personalized genome-scale metabolic modeling. Mol Syst Biol 10, 721 (2014). 


\section{Figure legends}

Figure 1: Effect of HFD and CMA treatment on liver histology, liver weights and body weight of Golden Syrian hamsters. (a) Study design. (b) Steatosis score of histological changes in liver. (c-f) Histological analysis of steatosis in liver sections stained with H\&E of liver. (g) Liver weight. (h) Body weight. (i) Lean / fat mass ratio. (j) The hepatic expression of $\mathrm{NAD}^{+}$. Control, normal diet; HFD, high-fat diet; HFD+MI_D1, high-fat diet supplemented with CMA at Dose I; HFD+MI_D2, high-fat diet supplemented with CMA at Dose II.

Figure 2: Effects of HFD and CMA on liver transcriptomics of Golden Syrian hamsters. (a) PCA plot of 37 hamsters. (b) Up- and down-regulated genes in HFD group as compared to control group. (c) KEGG pathway analysis shows pathways that were significantly altered between HFD and control groups, HFD+MI_D2 and HFD groups, respectively. Pathways that are down-regulated or up-regulated are shown in blue and red, respectively. The size of the bubble is proportional to $-\log _{10}$ of the FDR for each KEGG pathway term. (d) Up- and downregulated genes in HFD+MI_D2 group as compared to HFD group. (e) The log 2-fold changes in differential expressed genes between two groups compared to HFD group is significantly positive correlated.

Figure 3: Reporter metabolites in CMA-treated group with Dose II. Metabolic differences between the hamsters treated with or without CMA at Dose II were investigated through a comparative analysis of the gene expression profiles (RNA-seq) of the liver and iHamsterHepatocyte1818. (a) Liver-specific genome-scale metabolic model for Golden Syrian hamster was created using the Golden Syrian hamster orthologs of mouse genes based on the MMR. (b) reporter metabolites associated with amino acid metabolism and TCA cycle; (c) fatty acid activation and oxidation phosphorylation; (d) biosynthesis and metabolism of cholesterol, biosynthesis and elongation of fatty acid, and nucleotide metabolism. p values for each reporter metabolite were calculated for up- and downregulated genes. Abbreviations: MMR, mouse metabolic reaction; INIT, Integrative Network Inference for Tissue. 
Figure 4: Significant increases (red) and decreases (blue) in genes and associated reporter metabolites in liver from hamsters in HFD+MI_D2 as compared to of those in HFD group. (a) Reactions involved in one-carbon metabolism and transsulfuration pathway. (b) A summary of significant diffident genes involved in one-carbon metabolism, transsulfuration pathway, fatty acid metabolism, oxidative phosphorylation (OXPHOS), BCAAs degradation, TCA cycle and urea cycle. $*$ demonstrates the significance (adjusted p-value $<0.1$ ). (c) Reactions involved in fatty acid metabolism in both mitochondria and peroxisome. Abbreviations: $3-P G$, 3phosphoglycerate; $P H G D H$, phosphoglycerate dehydrogenase; 3-PSer, 3-phosphoserine; THF, tetrahydrofolate; SHMT2, serine hydroxymethyltransferase, mitochondrial; MTHFD1, C1THF synthase; GNMT, glycine N-methyltransferase; $D M G$, dimethylglycine; SARDH, sarcosine dehydrogenase; $D M G D H$, dimethylglycine dehydrogenase; BHMT2, Smethylmethionine--homocysteine S-methyltransferase BHMT2; CBS, cystathionine betasynthase; $C T H$, cystathionine gamma-lyase; $G S H$, glutathione; $G S S G$, glutathione disulfide; $G P X 1$, glutathione peroxidase 1; GPX4, phospholipid hydroperoxide glutathione peroxidase; $S A M$, S-Adenosyl methionine; PRDX5, peroxiredoxin-5, mitochondrial; $R-S-G S H$, S(formylmethyl)glutathione; SLC27A5, bile acyl-CoA synthetase; $A B C D 1$, ATP-binding cassette sub-family D member 1; ACOT8, Acyl-coenzyme A thioesterase 8; ACSM1, Acylcoenzyme A synthetase ACSM1, mitochondrial; ACSM3, Acyl-coenzyme A synthetase ACSM3, mitochondrial; ACSM5, Acyl-coenzyme A synthetase ACSM5, mitochondrial; $A C S S 2$, Acetyl-coenzyme A synthetase, cytoplasmic; ACSS3, Acyl-CoA synthetase shortchain family member 3, mitochondrial; ASS1, argininosuccinate synthase; CPS1, carbamoylphosphate synthase [ammonia], mitochondrial; MPV17L, mpv17-like protein; GSTK1, glutathione S-transferase kappa 1; COX7C, cytochrome c oxidase subunit 7C; ATP6V0E, vtype proton ATPase subunit e 1; ATP5E, ATP synthase subunit epsilon; ALDH2, aldehyde dehydrogenase, mitochondrial; $A L D H 9 A 1$ : 4-trimethylaminobutyraldehyde dehydrogenase; $B C A T 2$, branched-chain-amino-acid aminotransferase, mitochondrial; BCKDHA, 2oxoisovalerate dehydrogenase subunit alpha, mitochondrial; GPT2, alanine aminotransferase 
2; HOGA1, 4-hydroxy-2-oxoglutarate aldolase, mitochondrial; $I D H 2$, isocitrate dehydrogenase [NADP] , mitochondrial; MCCC1, methylcrotonoyl-CoA carboxylase subunit alpha, mitochondrial; $M C C C 2$, methylcrotonoyl-CoA carboxylase beta chain, mitochondrial; $P C C A$, propionyl-CoA carboxylase alpha chain, mitochondrial; $P R O D H$, proline dehydrogenase 1, mitochondrial; $S D H B$, succinate dehydrogenase [ubiquinone] iron-sulfur subunit; GOT1, aspartate aminotransferase, cytoplasmic; GPT, alanine aminotransferase 1; UQCRQ, cytochrome b-c1 complex subunit 8; UQCR11 : cytochrome b-c1 complex subunit 10; SLC6A12, sodium- and chloride-dependent betaine transporter; SLC43A1, large neutral amino acids transporter small subunit 3; $S D H B$, succinate dehydrogenase [ubiquinone] iron-sulfur subunit; NDUFS7, NADH dehydrogenase [ubiquinone] iron-sulfur protein 7; NDUFB7, $\mathrm{NADH}$ dehydrogenase [ubiquinone] 1 beta subcomplex subunit 7; NDUFA13, NADH dehydrogenase [ubiquinone] 1 alpha subcomplex subunit 13; ND3, NADH-ubiquinone oxidoreductase chain $3 ; F A s$, fatty acids.

Figure 5: (a) Reactions involved in BCAAs degradation, tricarboxylic acid cycle, and urea cycle. (b) Global effect of CMA supplementation. Abbreviations: $O A A$, Oxalacetic acid; $\alpha-K G$, alpha-ketoglutarate; $H O G, 4-$ hydroxy-2-oxoglutarate; $M$-CoA, methylmalonyl-CoA; P5C, 1-pyrroline-5-carboxylate; $H c y$, homocysteine, $N R$, nicotinamide riboside; $N A C$, N-acetyl-1cysteine; FAs, fatty acids; $L C T$, L-carnitine tartrate. 
Table 1: Effects of HFD and multi-ingredients treatment on biometric and serum variables.

\begin{tabular}{|c|c|c|c|c|}
\hline & $\begin{array}{c}\text { Control } \\
(\mathrm{n}=9)\end{array}$ & $\begin{array}{c}\text { HFD } \\
(\mathbf{n}=\mathbf{1 0})\end{array}$ & $\begin{array}{l}\text { HFD + MI_D1 } \\
\quad(\mathbf{n}=\mathbf{1 0})\end{array}$ & $\begin{array}{l}\text { HFD + MI_D2 } \\
\quad(n=10)\end{array}$ \\
\hline \multicolumn{5}{|l|}{ Biometric variables } \\
\hline $\begin{array}{l}\text { Initial body weight }(\mathrm{g}) \\
\text { Final body weight }(\mathrm{g})\end{array}$ & $\begin{array}{l}123.05 \pm 5.14 \\
122.20 \pm 5.62\end{array}$ & $\begin{array}{c}121.07 \pm 7.54 \\
126.57 \pm 8.20^{\mathrm{d}}\end{array}$ & $\begin{array}{c}119.85 \pm 7.51 \\
120.08 \pm 10.32\end{array}$ & $\begin{array}{c}122.55 \pm 6.90 \\
116.28 \pm 9.86^{\mathrm{cf}}\end{array}$ \\
\hline Liver weight (g) & $4.23 \pm 0.30$ & $5.14 \pm 0.65^{\mathrm{a}}$ & $4.69 \pm 0.60$ & $4.62 \pm 0.61$ \\
\hline Liver weight (\%) & $3.49 \pm 0.25$ & $4.04 \pm 0.29^{\mathrm{a}}$ & $3.92 \pm 0.43$ & $3.98 \pm 0.29$ \\
\hline Initial fat mass $(\%)$ & $11.94 \pm 3.20$ & $13.87 \pm 1.95$ & $14.40 \pm 3.12$ & $14.04 \pm 2.28$ \\
\hline Final fat mass $(\%)$ & $11.28 \pm 2.60$ & $13.14 \pm 2.78$ & $14.84 \pm 4.19$ & $13.10 \pm 2.87$ \\
\hline Initial lean mass (\%) & $84.89 \pm 3.26$ & $84.27 \pm 1.94$ & $84.06 \pm 2.99$ & $84.02 \pm 2.20$ \\
\hline Final lean mass (\%) & $85.42 \pm 2.40$ & $85.74 \pm 3.03$ & $83.95 \pm 4.04$ & $85.22 \pm 3.09^{\mathrm{f}}$ \\
\hline Initial lean/fat mass ratio & $7.83 \pm 3.22$ & $6.20 \pm 1.01$ & $6.21 \pm 1.97$ & $6.16 \pm 1.22$ \\
\hline Final lean/fat mass ratio & $8.00 \pm 2.13$ & $6.84 \pm 1.67$ & $6.20 \pm 2.19$ & $6.83 \pm 1.70$ \\
\hline Cumulative food intake (kcal) & $126.77 \pm 14.43$ & $111.08 \pm 13.22^{\text {ad }}$ & $104.22 \pm 15.94^{\mathrm{e}}$ & $95.23 \pm 10.69^{\mathrm{cf}}$ \\
\hline \multicolumn{5}{|l|}{ Plasma variables } \\
\hline $\begin{array}{l}\text { Glucose (mM) } \\
\text { Insulin (mIU/L) }\end{array}$ & $\begin{array}{c}7.29 \pm 1.50 \\
16.69 \pm 2.92\end{array}$ & $\begin{array}{l}9.43 \pm 2.01^{\mathrm{a}} \\
16.26 \pm 3.61\end{array}$ & $\begin{array}{c}8.93 \pm 1.42 \\
16.17 \pm 1.34\end{array}$ & $\begin{array}{c}9.27 \pm 2.01 \\
16.78 \pm 3.43\end{array}$ \\
\hline $\mathrm{CHOL}(\mathrm{mM})$ & $2.87 \pm 0.21$ & $4.03 \pm 0.65^{\mathrm{a}}$ & $3.77 \pm 0.60$ & $4.44 \pm 0.85$ \\
\hline HDL-C (mM) & $2.57 \pm 0.37$ & $3.15 \pm 0.50^{\mathrm{a}}$ & $2.88 \pm 0.52$ & $3.32 \pm 0.60$ \\
\hline LDL-C (mM) & $0.90 \pm 0.20$ & $1.38 \pm 0.33^{\mathrm{a}}$ & $1.22 \pm 0.24$ & $1.34 \pm 0.28$ \\
\hline $\mathrm{TG}(\mathrm{mM})$ & $1.07 \pm 0.30$ & $1.15 \pm 0.53$ & $0.96 \pm 0.31$ & $0.82 \pm 0.32$ \\
\hline RWAT (\%) & $1.03 \pm 0.23$ & $0.87 \pm 0.17$ & $0.99 \pm 0.24$ & $0.90 \pm 0.13$ \\
\hline MWAT (\%) & $0.88 \pm 0.24$ & $1.21 \pm 0.24^{\mathrm{a}}$ & $1.17 \pm 0.29$ & $1.14 \pm 0.19$ \\
\hline MUS & $0.27 \pm 0.03$ & $0.25 \pm 0.03$ & $0.24 \pm 0.03$ & $0.24 \pm 0.03$ \\
\hline
\end{tabular}

Data are showed as the mean \pm SD. Different superscript letters indicate statistically significant differences $(p<0.05$, Student' $t$-test). a, b and $c$ indicate difference between HFD and control groups; between HFD+MI_D1 and HFD group; between HFD + MI_D2 and HFD groups, respectively. d, e and f show difference between 8 weeks (initial) and 12 weeks (final) in HFD, HFD+MI D2 and HFD+MI D2 group, respectively. RWAT, retroperitoneal white adipose tissue. MWAT, mesenteric white adipose tissue. MUS, gastrocnemius and soleus muscles. 


\section{Supplementary datasets}

Dataset S1: List of genes identified in liver transcriptomics and DEGs comparing HFD to control, HFD+MI_D1 to HFD, HFD+MI_D2 to HFD, and HFD+MI_D1 to HFD+MI_D2, respectively.

Dataset S2: KEGG pathway and GO enrichment analysis for DEGs in HFD versus control and HFD+MI_D2 versus HFD.

Dataset S3: Reporter metabolites are presented using the differential expression pf genes in the liver tissue of the CMA-treated hamsters compared with that of the liver tissue of the HFD hamsters.

Dataset S4: Composition of the diets used in the study. 
A

\section{MMR}

8,140 reactions

3,579 genes

5,992 metabolites

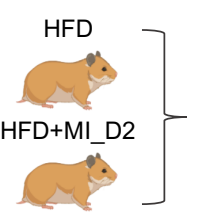

B

\section{Hamster orthologs iHamsterHepatocyte1818} of mouse genes

INIT

Transcriptomics
3,867 reactions

,818 genes

3,205 metabolites

Reporter metabolite

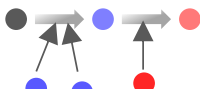

$T_{0}^{1} T$

Up
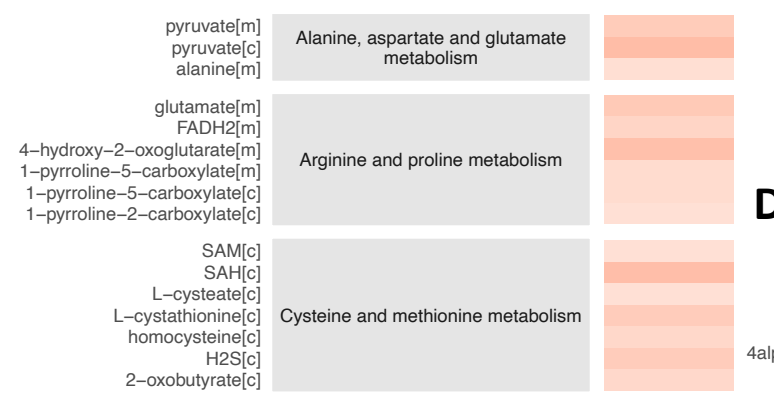

pentaglutamyl-folate(THFF[][c]

pentaglutamyl-folate(DHF)[m]
NADPH[c]

hexaglutamyl-folate(THF)[c]

heptaglutamyl-folate(THF)

heptaglutamyl-folate(DHF)

Folate metabolism

CPMP[C
$5,10-$ methylene-THF[m]
$10-$ formyl-THF[c]
$10-$ or

10-formyl-THF-glu(6):

THF-hexaglutamate[c]

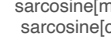

formaldehyde[m

formaldehyde[ $[\mathrm{m}$

dimethylglycinel $[\mathrm{c}$

Glycine, serine and threonine

phenylpyruvate|c
L-formykynurenine

homogentisate

anthranilate

4alpha-hydroxytetrahydrobiopterin
4-maleylacetoacetate

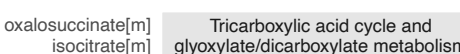

4-methyl-2-oxopentanoate[m]
3-methyl-2-oxobutyrate[m]
$2-0 x 0-3-$ methyyvalerate $[m]$$\quad \begin{gathered}\text { Valine, leucine, and isoleucine } \\ \text { metabolism }\end{gathered}$

Reporter metabolites $\begin{gathered}\text { Subsystems in } \\ \text { iHamsterHepatocyte1818 }\end{gathered}$

-log( $p$-value)

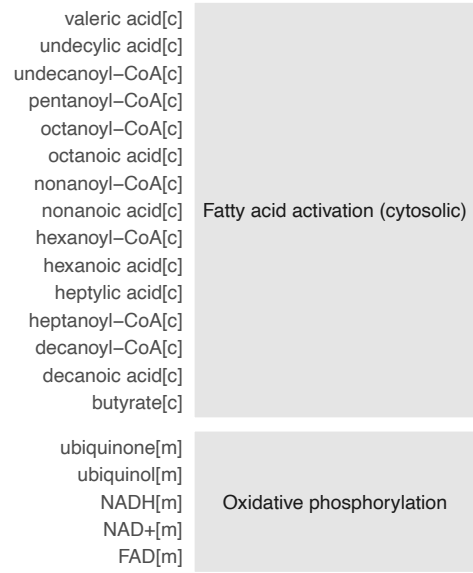

Reporter metabolites $\quad \begin{gathered}\text { Subsystems in } \\ \text { iHamsterHepatocyte1818 }\end{gathered}$

Down

5alpha-cholesta-8,24-dien-3-one[c]

(Bloch pathway) 3-keto-4-methylzymosterol[c] (R)-mevalonate[c]

4alpha-methyl-5alpha-cholesta-8-en-3-one[c] 4alpha-carbory-4bera-my-5apha-cholesta-8-en $3-0 n e[c]$

Cholesterol biosynthesis 3
(Kandustch-Russell pathway)

\begin{tabular}{|c|c|}
\hline $\begin{array}{r}\text { HMG-CoA[c] } \\
\text { acetyl-CoA[c] } \\
\text { acetoacetyl-CoA[c] }\end{array}$ & Cholesterol metabolism \\
\hline 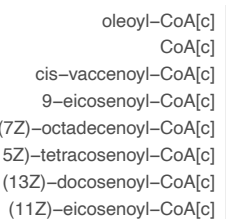 & Fatty acid biosynthesis (unsaturated) \\
\hline $\begin{array}{r}\text { tetracosanoyl-CoA }[c] \\
\text { stearoyl-CoA[c] } \\
\text { palmitoyl-CoA }[\mathrm{c}] \\
\text { eicosanoyl-CoA }[\mathrm{c}] \\
\text { docosanoyl-CoA[c] }\end{array}$ & Fatty acid elongation (even-chain) \\
\hline $\begin{array}{r}\text { nonadecanoyl-CoA[c] } \\
\text { heptadecanoyl-CoA[c] } \\
\text { heneicosanoyl-CoA[c] }\end{array}$ & Fatty acid elongation (odd-chain) \\
\hline $\begin{array}{r}\text { UTP[c] } \\
\text { uridine[s] } \\
\text { uridine[[] } \\
\text { UMP[I] } \\
\text { cytidine[[] } \\
\text { CMP[I] } \\
\text { AMP[I] } \\
\text { adenosine[[]] }\end{array}$ & Nucleotide metabolism \\
\hline
\end{tabular}




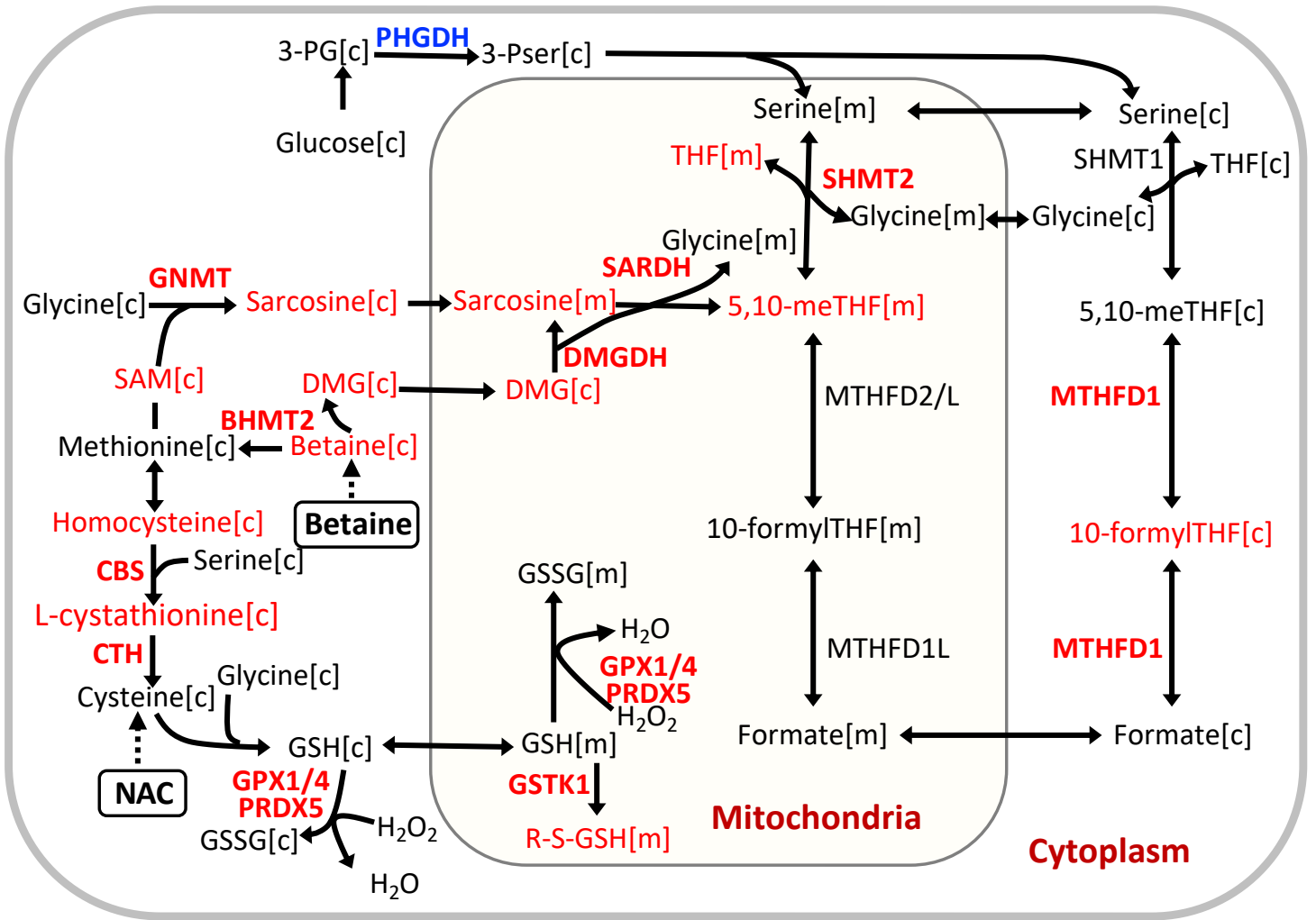

Log2FC

UQCRQ -

UQCR11 -

SLC43A1 -

SLC27A5 -

SHMT2 -

SDHB -

PRODH -

PRDX5 -

PHGDH -

NDUFS7 -

DIUFB -

NDUFA13 -
ND3 -

MTHFD1 -

MCCC 1 -

IDH2 -

HOGA1 -

GPX4 -

GPX1 -

GPT

GOT1 -
GNMT -

DMGDH -

CTH -

COX7C -

CBS -
BHMT2 -

BCKDHA -

BCAT2 -

ATP6V0E -

ATP5E -

ASS1 -

LDH9A1 -

ACSM3 -

ACSM1 -

ACOT8 -

$\mathrm{ABCD} 1$ -

C

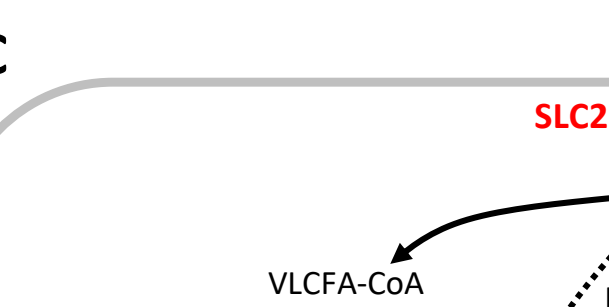

FAs

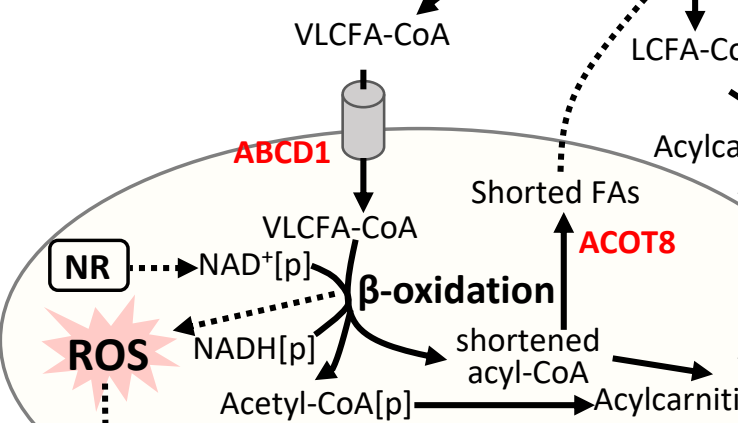

FAs pool $\cdots$ L-carhitine[c]
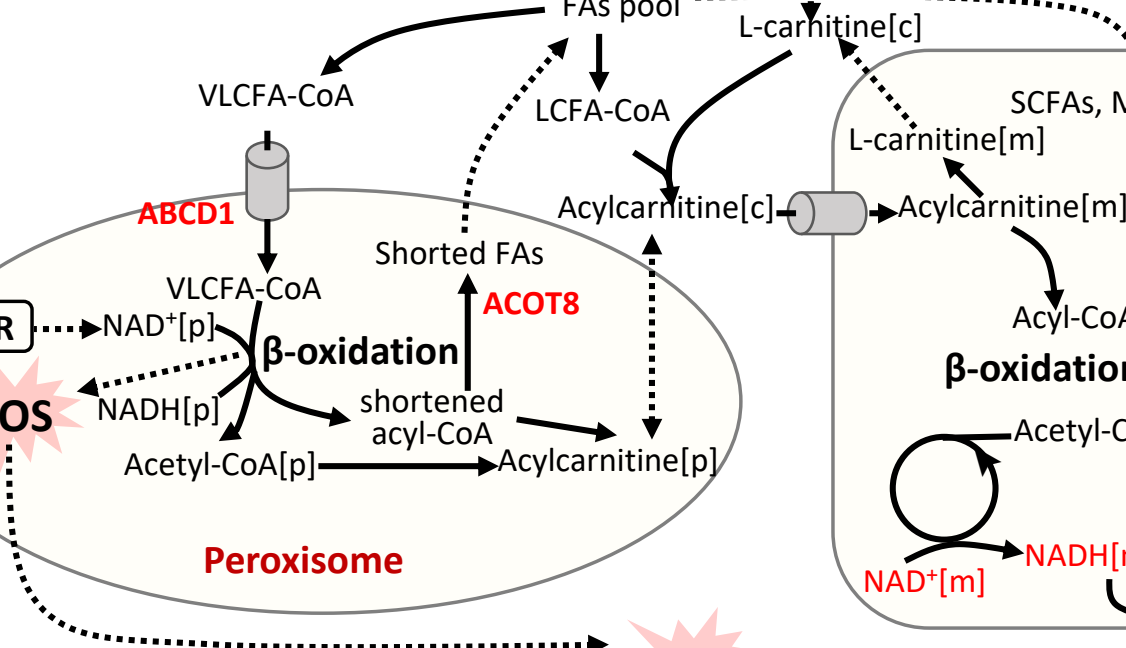

ROS

SCFAs, MCFAs

Mitochondria

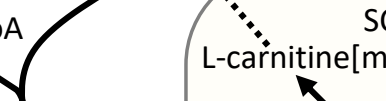

L-carnitine $[\mathrm{m}] \quad$ ACSM1

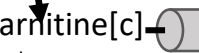

Acylcarnitine $[\mathrm{m}] \quad \begin{aligned} & \text { ACSM3 } \\ & \text { ACSM5 } \\ & \text { ACSS2 }\end{aligned}$

ACSSS
ACSS3

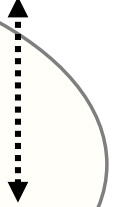

Acyl-CoA[m] NAD ${ }^{+}[\mathrm{m}]$ 4... NR 


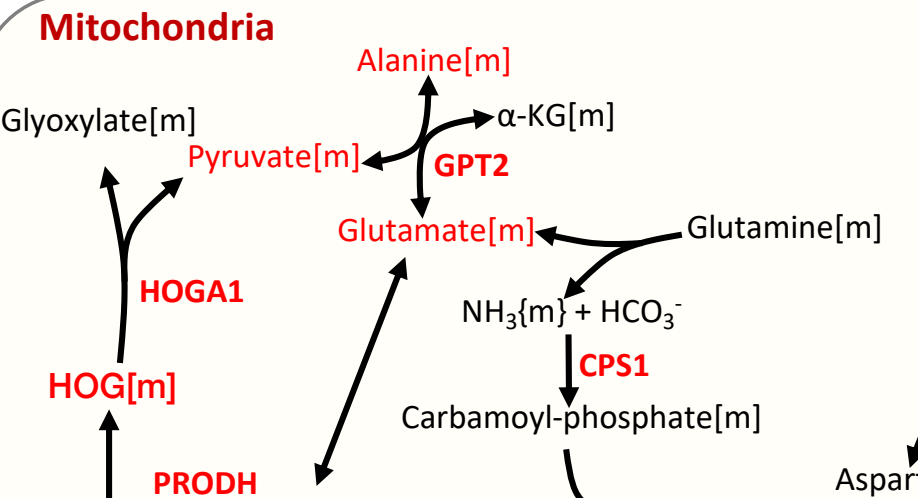

Valine, leucine and isoleucine degradation

Proline $[\mathrm{m}] \longleftrightarrow \mathrm{P} \longmapsto \mathrm{C}[\mathrm{m}] \longleftrightarrow$ Ornithine $[\mathrm{m}] \longrightarrow$ Citrulline[m]

Arginine and
proline metabolism Ornithine[c] Citrulline[c]

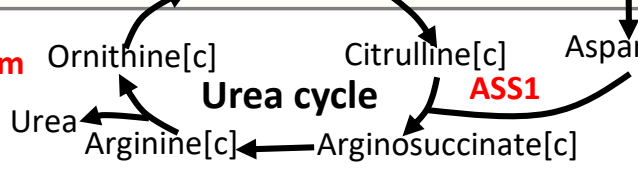

Arginine biosynthesis

Cytoplasm

Aspartate[m] SDHB Succihate[m]

BCAT2

BCKDHA

\begin{tabular}{l|l} 
MCCC1 \\
MCCC2 \\
Acetyl-CoA[m]
\end{tabular} $\begin{aligned} & \text { ALDH2 } \\
& \text { ALDH9A1 } \\
& \text { Propanoyl-CoA[m] }\end{aligned}$

OAÁ[m] Isocitrate[m] $\downarrow$

1 TCA cycle fIDH2 $\mathrm{M}-\mathrm{COA}[\mathrm{m}]$

Malate $[\mathrm{m}]$
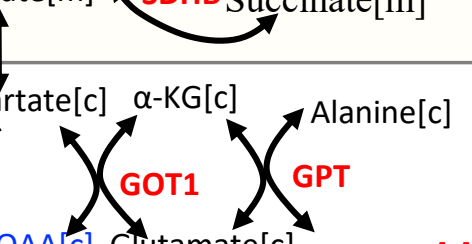

OAA[c] Glutamate[c] Pyruvate[c]

B

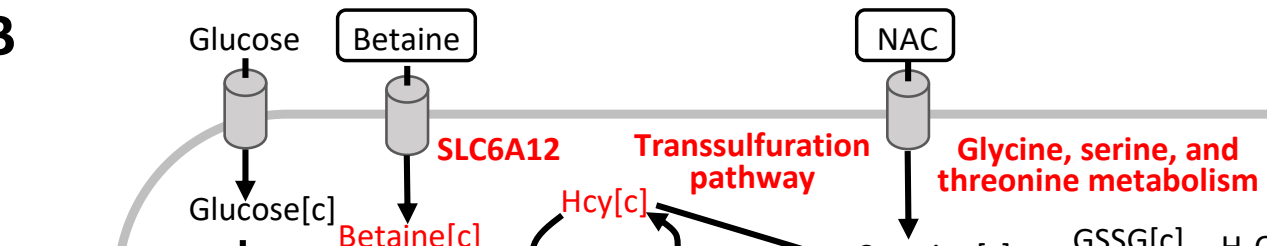

BCAAs

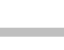

